\title{
COMPETENCIAS CIENTÍFICAS Y RESOLUCIÓN DE PROBLEMAS EN EL INSTITUTO PEDAGÓGICO NACIONAL (IPN)
}

\author{
Ricardo Andrés Franco frankoupn@gmail.com ${ }^{1}$
}

\section{Resumen:}

En este documento se presentan los resultados del proyecto de Práctica Pedagógica y Didáctica, de Licenciatura en Química en el Instituto Pedagógico Nacional, en donde se implementó una cartilla de actividades para el grado décimo y centrada en la resolución de problemas, dando cuenta del desarrollo de competencias científicas.

Palabras Clave

Resolución de Problemas;

Cartilla de Actividades;

Competencias Científicas 


\section{Introducción}

La formación de profesores dentro del Proyecto Curricular Experimental Para la Formación de Licenciados en Química de la Universidad Pedagógica Nacional, se concibe como un proceso de investigación y reflexión alrededor de la Práctica Pedagógica y Didáctica $(P$. $P$ y D), a la vez que esta se asume como un escenario de formación, donde se posibilita la construcción de una escuela de pensamiento, en la que tanto, estudiantes como profesores, reflexionen y transformen la realidad educativa; investigando, experimentando e innovando sobre los problemas y realidades de la educación en ciencias.

Así, se ha desarrollado un proyecto de P.P y D en el Instituto Pedagógico $\mathrm{Na}-$ cional de la Ciudad de Bogotá durante el año 2008. En la primera fase de la P. P y D: la caracterización, se auscultó el pensamiento de un profesor de educación media, en relación con sus concepciones sobre Química y su enseñanza. A partir de tales indagaciones se diseñó una estrategia de intervención centrada en la resolución de problemas para el desarrollo de competencias científicas: la cartilla de actividades, siendo el marco conceptual y metodológico, el desarrollo de competencias científicas investigativas (Chona, Arteta, Fonseca, Martínez \& lbáñez, 2008) y la resolución de problemas (García, 2000; Gil Pérez, 1998). A su vez, el trabajo se inscribe en la línea de investigación sobre el pensamiento del profesor (Marcelo, 2002).

\section{Marco de Referencia}

El estudio del desarrollo de competencias científicas por parte de los profesores, se inscribe en la línea de investigación sobre el pensamiento del profesor, en el sentido de que, es en la mente del profesor en donde ocurren los procesos que organizan y dirigen su conducta. De acuerdo con L. Reyes y L. E Salcedo (1998), esta línea surge como respuesta a la necesidad de consolidar procesos diferentes para la enseñanza y el aprendizaje de las ciencias, a la vez que emerge como un espacio de exploración, reflexión, análisis y construcción de conocimientos en 
torno a las relaciones entre las acciones de los profesores de ciencias. De otra parte, en el análisis de la relación pensamiento-acción del profesor, las influencias del conocimiento, las creencias, los valores $\mathrm{y}$ las destrezas desempeñan un papel preponderante, aún en fases iniciales de su formación (Marcelo 2002).

Respecto a las competencias científicas existen varias apreciaciones. Por una parte, estas se configuran en la capacidad de un sujeto, expresada en desempeños observables y evaluables ,que evidencia formas sistemáticas de razonar y explicar el mundo natural y social, a través de la construcción de interpretaciones apoyadas por los conceptos de las ciencias (Chona et., al, 2008), a la vez que representan la capacidad que posee un estudiante para hacer uso de sus conocimientos y habilidades para lograr un propósito determinado (Cárdenas, 1998).

\section{Metodología}

A partir de los resultados obtenidos en la primera fase de caracterización en la P. P. y $D$, se explicitó la imagen de ciencia que posee un profesor en ejercicio, y su imagi-
En este marco, el desarrollo de competencias científicas en los estudiantes en parte ha sido posible mediante la resolución de problemas. Un problema, de representa entonces una situación para cuyas dificultades no hay soluciones a la vista (Gil Pérez, 1998), Así, la resolución de situaciones-problema se ha consolidado como una importante opción metodológica para la enseñanza de las ciencias, cuyos resultados se traducen en la construcción de un cuerpo de conocimientos científicamente argumentado, en el sentido de que la resolución de problemas permite, por una parte, que los estudiantes pongan en práctica el conocimiento químico en particular, y contribuye a cambiar sus actitudes hacia la ciencia. Puntualícese que entre resolver problemas y responder ejercicios de lápiz y papel existen amplias diferencias (García, 2000).

nario, el cual incide en el desarrollo de competencias científicas en los estudiantes, práctica ésta que puso de manifiesto la necesidad de diseñar una estrategia didáctica centrada en la resolución de 
problemas, que contribuyera al desarrollo de dichas competencias, posibilitando también la reflexión conjunta con el profesor titular, de conformidad con las posibilidades de cambio y mejoramiento allí señaladas.

Para la fase de intervención se diseñó e implementó una la cartilla de actividades como estrategia didáctica centrada en la resolución de problemas hacia el desarrollo de competencias científicas en estudiantes de grado décimo.
Para cada Fase Temática se propuso un desarrollo conceptual y metodológico, donde los conceptos científicos se abordaron alrededor de un entorno cotidiano, cuyas aplicaciones fueron evidentes en la resolución de problemas. La estructura de dicha cartilla se centró en lecturas científicas de actualidad, un video clip, una lectura sobre la temática específica, y actividades constituidas por situaciones problema. Además del análisis de las cartillas de actividades, se tuvo en cuenta la opinión de varios estudiantes acerca de la estrategia.

\section{Resultados y discusión}

Se elaboraron diez actividades, incluyendo dos de laboratorio y una evaluación bimestral; de cada actividad se ha seleccionado una situación problema, y se ha analizado su resolución escogiendo una cartilla por cada equipo de trabajo en clase, y siguiendo una categorización por etapas del desarrollo de un problema (García, 2000). En este marco, las etapas de la resolución de problemas para 10 Situaciones distintas durante la implementación, son: A-Lectura previa del problema. B-Comprensión del problema. C-Análisis de los elementos del problema. $\boldsymbol{D}$ Comprobación del problema. E-Selección de datos relevantes. $\boldsymbol{F}$ -Consulta de lo que no se sabe. G-Selección de métodos adecuados. H-Resolución del problema. 


\section{$\underline{\text { Tabla } 1}$}

Desempeños de los estudiantes en las etapas de resolución de las situaciones en la cartilla de actividades

\begin{tabular}{|c|c|c|c|c|c|c|c|c|c|c|}
\hline $\begin{array}{c}\text { Situaciones } \\
\text { Problema }\end{array}$ & 1 & 2 & 3 & 4 & 5 & 6 & 7 & 8 & 9 & 10 \\
\hline Etapa & \multicolumn{10}{|c|}{ Número de equipos de trabajo que presentan desempeño } \\
\hline A & 6 & 8 & 7 & 7 & 8 & 5 & 8 & 8 & 7 & 8 \\
\hline B & 1 & 3 & 6 & 5 & 7 & 4 & 6 & 7 & 7 & 5 \\
\hline C & 3 & 4 & 3 & 4 & 7 & 3 & 6 & 6 & 5 & 6 \\
\hline D & 2 & 4 & 4 & 3 & 6 & 1 & 6 & 7 & 7 & 4 \\
\hline E & 4 & 6 & 5 & 5 & 7 & 4 & 7 & 7 & 6 & 3 \\
\hline F & 1 & 3 & 4 & 3 & 6 & 4 & 6 & 8 & 7 & 6 \\
\hline G & 2 & 3 & 7 & 3 & 6 & 2 & 5 & 7 & 6 & 6 \\
\hline H & 1 & 2 & 6 & 2 & 5 & 2 & 5 & 7 & 6 & 6 \\
\hline
\end{tabular}

Las situaciones problema son:

- El Hipercentro de Materiales

- Las Almas Benditas: ¿Mito o realidad?

- Modelos atómicos: ¿de la noche a la mañana?

- David perdió su libreta de apuntes

- Atentado al Club el Nogal

- Entrevista de Trabajo...

- Un estudiante curioso se envenenó...

- El caso del Perro Mateo

- Qué Viaje...

- Latonero confundido...

Así, la cartilla de actividades se ha dividido en tres momentos: inicial, intermedio y final. 
Como se puede apreciar, (Tabla 1) los desempeños observables de los estudiantes con respecto al momento inicial (situaciones problema $1 \mathrm{y}$ 2) se evidencian únicamente en la lectura previa del problema y en la selección de datos relevantes, encontrándose una mayor falta de desempeños en las etapas comprensión del problema, comprobación del problema y resolución del problema. Al respecto, Gil. Pérez (1998), señala que, los estudiantes novatos recurren a la manipulación inmediata de datos para que el profesor escuche de ellos datos precisos: "Pues... prácticamente todos los ejercicios de química son problemas... entonces, casi no hay diferencia..." Est. 1.

En este sentido, se evidencia una cultura científica centrada en el desarrollo de procedimientos de tipo algorítmico, coherente con el imaginario de personas poco familiarizadas con el tema, cuyas concepciones son difíciles de modificar (Gil Pérez, 1998). Sin embargo, en esta concepción de problema se reconoce una diferencia concreta entre ejercicio y problema: "Pues... prácticamente todos los ejercicios de química son problemas... entonces, casi no hay diferencia pero... de cierto modo un problema es algo a lo que uno le tiene que buscar solución, y un ejercicio es algo que ya tiene solución" Est 1.

Con relación al momento intermedio (situaciones 3-7), se presenta un crecimiento relativo de los desempeños en las diferentes etapas de resolución, lo que es coherente con opiniones divergentes de los estudiantes frente a las actividades: "es como muy ortodoxo, ya muy mecánico...es mejor algo como que uno pueda aplicar ¿si me entiendes?, como que también ver la química desde aquí desde nuestro lugar, desde ya, de nosotros, desde nuestro espacio" Est. 2. "Es más chévere porque es como más de actividad, y ubicarse en el lugar, eso ya cambia la situación. Pero eso de que defina acá de que defina tal cuál..." Est 3. 
Respecto al momento final (situaciones 8-10), hay un aumento marcado en todos los desempeños observables, que refleja la construcción de una cultura alrededor de la resolución de problemas, pues los desempeños en esta última etapa se incrementan: "...pues la cartilla de actividades nos ha servido como a entender un poco más, la química, y si tenemos algunos problemas, pues llegamos a resolverla con el profesor, y pues a comentarla con todo el salón": Est 4. Así, se presenta un aumento progresivo en los desempeños observables de los grupos en cada etapa de la resolución de problemas, cuya explicación deriva especialmente de la particularidad de las situaciones planteadas, que se enmarcaron dentro de una rigurosidad conceptual, pero a la vez llamativo en la medida de las posibilidades y actitudes de los estudiantes: "Eso fue algo más dinámico, o sea, fue algo diferente, porque digamos... mucha gente se animó a participar, digamos yo, que casi nunca pongo cuidado..." Est 5.

En este sentido, la resolución de problemas a partir de la puesta en prácti- ca de los conocimientos químicos se constituye en el eje articulador del desarrollo de estos procesos de pensamiento complejo: "Las aplicaciones en la vida real... situaciones que si digamos a uno le pasan, uno ya sabe cómo reaccionar a ese problema" Est 6. "Digamos... en un laboratorio tuvimos el ejemplo de un perro que consumía látex... y entonces teníamos... bueno... eso que son casos que pasan en la vida real... también tuvimos el caso del atentado al club el nogal, identificar los diferentes... procesos químicos que se daban en ese instante ¿si?..." Est 1.

\section{Apropiación del Cambio}

Durante la implementación de la cartilla de actividades, y en general en la fase de Intervención, se generó una interacción constante de los profesores en formación y titular, reflexiones que se evidencian en nuevas elaboraciones acerca del discurso de la didáctica de las ciencias, lo que contribuye a modificar algunos aspectos de la imagen de ciencia: "Considero que en las diferentes actividades de los seres humanos, podemos hablar de 
verdaderas competencias cientificas cuando nosotros sabemos hacer en un contexto, muchas veces confundimos el tener un conocimiento exacto de un determinado tema, y desafortunadamente nos quedamos solo en eso, y no logramos llegar a la etapa de saber desarrollar esa habilidad" Profesor 1.

\section{Consideraciones finales}

La implementación de la cartilla de actividades centrada en la resolución de problemas, se ha constituido en un importante recurso de apoyo como estrategia didáctica para la enseñanza de algunos conceptos químicos, propiciando así el desarrollo de competencias científicas, expresadas en capaci- dades de los estudiantes, para poner en práctica el conocimiento químico por medio de los procedimientos de las ciencias. Sin embargo, la enculturación científica cada vez se pone más en cuestión, problema que desde el punto de vista abordado, debe considerarse a partir del desarrollo de competencias básicas como interpretar, argumentar y proponer en química, a partir de habilidades como: leer, escribir, hablar y escuchar. Al respecto, surgen nuevos horizontes de investigación acerca de cómo diseñar e implementar estrategias didácticas para lograr el desarrollo de estas competencias básicas.

\section{Bibliografía}

Chona, G., Arteta, J., Fonseca, G., Martínez, S, Ibáñez, S. (2008). Informe del Proyecto de Investigación: El Desarrollo de Competencias Científicas Investigativas y su Relación con el Conocimiento Profesional de Profesores de Ciencias. Universidad Pedagógica Nacional- CIUP.

Cárdenas, F. 1998. Desarrollo y evaluación de los procesos de razonamiento complejo en ciencias. En: Revista TE $\Delta$ NN$^{\circ} 3$ de la Facultad de Ciencia y Tecnología. Universidad Pedagógica Nacional. Bogotá.

García, J. (2000). La solución de situaciones problemáticas: una estrategia didáctica para la enseñanza de la química.

Gil, Pérez. D. (1998). La Resolución de Problemas de Lápiz y Papel Como Actividad de Investigación. Investigación en la Escuela No 6, 1998. 

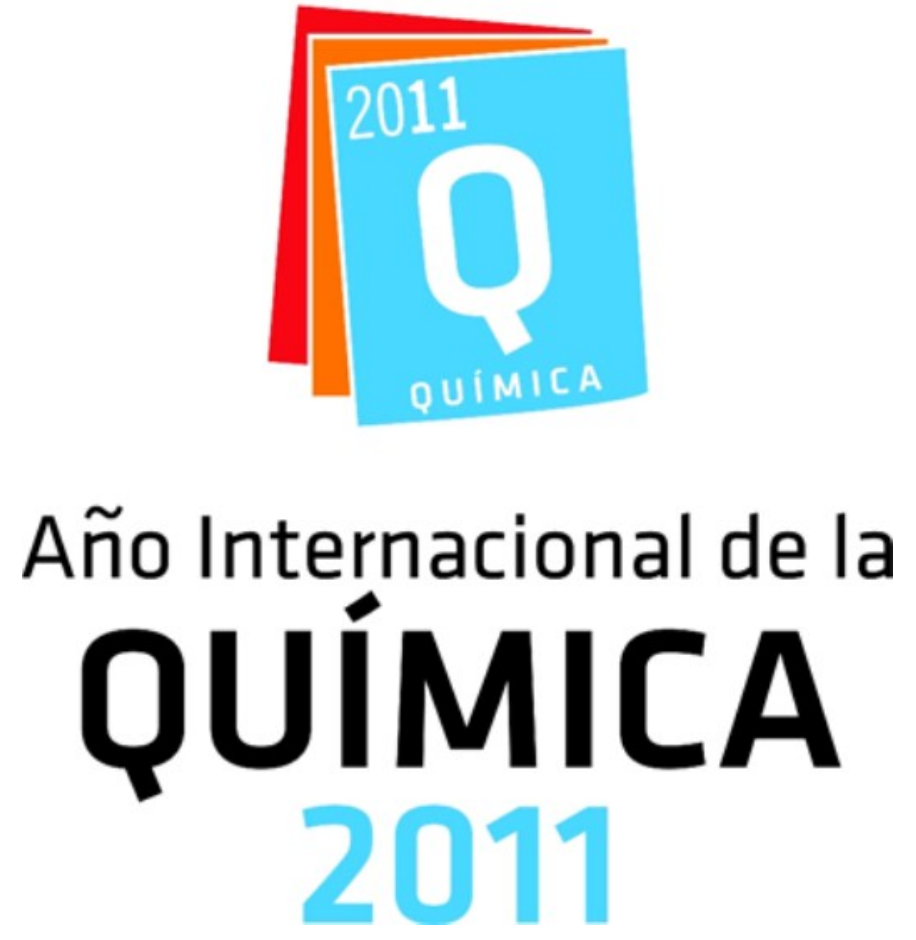